\title{
Evidencias de Validez Convergente y de Criterio del Maternal Behavior Q-Sort (MBQS) 3.1 en Madres Peruanas
}

\author{
Convergent and Criterion Validity of the Maternal Behavior Q-Sort 3.1 in a Sample \\ of Peruvian Mothers
}

\author{
Patricia Bárrig-Jó ${ }^{1}$ Magaly Nóblega ${ }^{2}$ y Andrea Ugarte $^{3}$
}

\begin{abstract}
Resumen
El presente estudio presenta evidencias de validez convergente del MBQS 3.1 con la versión 2.1 y de validez de criterio con el Attachment Q-Sort (AQS). La muestra estuvo conformada por 27 madres peruanas con hijos de ocho a once meses de edad y de nivel socioeconómico bajo de Lima. La edad promedio fue de 27.96 años $(D E=6.02)$ y la mayoría reportó estudios secundarios $(63 \%)$. Se encontró una fuerte y positiva asociación entre las versiones 2.1 y $3.1(r=.76, p<.01)$. Los perfiles de conducta materna del MBQS 3.1, sensible, conectada y sincrónica, correlacionaron entre sí con valores mayores a $.90(p<.01)$. Finalmente, se obtuvo una correlación de $.44(p<.05)$ con el AQS. El MBQS 3.1 muestra adecuados índices de validez lo cual resulta favorable para su uso en la medición de la sensibilidad materna en el contexto peruano.
\end{abstract}

Palabras clave: sensibilidad materna, MBQS, validez convergente, validez de criterio, infantes

\begin{abstract}
The Maternal Behavior Q-Sort (MBQS) assesses the maternal sensitivity of mothers of 0-3 year-olds in natural contexts. This study presents the results of MBQS 3.1 convergent validity with version 2.1 and criterion validity with the Attachment Q-Sort (AQS). The sample was comprised of 27 Peruvian mothers with children between 8 and 11 months old, from a low socioeconomic sector in Lima. The mean age of the mothers was 27.96 years $(S D=6.02)$ and most reported at least some high school education $(63 \%)$. The maternal behavior profiles in MBQS 3.1 - sensitive, connected, and synchronic - correlated with values higher than .90 ( $p<.01)$. A strong positive association was found between versions 2.1 and $3.1(r=.76, p<.01)$. Finally, a correlation of $.44(p<.05)$ was obtained with AQS. The MBQS 3.1 shows adequate validity indicators, which recommend its use for the measurement of maternal sensibility in Peru.
\end{abstract}

Keywords: maternal sensitivity, MBQS, convergent validity, criterion validity, infants

\footnotetext{
${ }^{1}$ Doctora en Psicología por la University of Vermont (UVM). Docente asociada y miembro del Grupo de Investigación Relaciones Vinculares y Desarrollo Socioemocional, Departamento de Psicología, PUCP. Pontificia Universidad Católica del Perú. Av. Universitaria 1801, San Miguel, 15088, Lima, Perú. Tel.: 51-1-6262000 anexo: 4587. Correo: pbarrig@ @ucp.pe

${ }^{2}$ Doctora en Psicología por la Pontificia Universidad Católica del Perú (PUCP). Docente Principal y Coordinadora del Grupo de Investigación Relaciones Vinculares y Desarrollo Socioemocional, Departamento de Psicología, PUCP. Pontificia Universidad Católica del Perú. Av. Universitaria 1801, San Miguel, 15088, Lima, Perú. Tel.: 51-1-6262000 anexo: 4534. Correo: mnoblega@pucp.pe

${ }^{3}$ Licenciada en Psicología Clínica por la Pontificia Universidad Católica del Perú (PUCP). Asistente de Docencia y miembro del Grupo de Investigación Relaciones Vinculares y Desarrollo Socioemocional, Departamento de Psicología, PUCP. Pontificia Universidad Católica del Perú. Av. Universitaria 1801, San Miguel, 15088, Lima, Perú. Tel.: 51-1-6262000 anexo: 4534. Correo: andrea.ugarte@ pucp.pe 


\section{Introducción}

La teoría del apego sostiene que el lazo emocional entre un infante y su cuidador principal o figura de apego, que por lo general es la madre, se construye a partir de las experiencias e interacciones entre ambos (Bowlby 1969/1982; Marrone, 2001). En sus estudios de la interacción madre-infante en Uganda (Ainsworth, 1967) y en el Procedimiento de la Situación del Extraño en Baltimore (Ainsworth, Bell, \& Stayton, 1971), Ainsworth observó que la calidad de la respuesta materna promovería la seguridad del niño/a en situaciones de separación y retorno. Los patrones de comportamiento observados fueron la base para configurar tres tipos de apego: seguro, evitativo y ambivalente (Ainsworth, Blehar, Waters, \& Wall, 1978), y posteriormente se introdujo la categoría de apego desorganizado (Main \& Solomon, 1990). Respecto al comportamiento materno, Ainsworth (1969) acuñó el término de sensibilidad para aludir a la capacidad para percibir, interpretar y responder adecuadamente a las demandas emocionales, sociales y físicas del bebé o del niño(a) (Bretherton, 2013). Esto supone una habilidad para adaptarse a las propias necesidades y las del niño/a, en una interacción cálida y armoniosa (Marrone, 2001). Así, identificó cuatro ejes importantes de esta capacidad: 1) la conciencia de las señales, para que éstas puedan ser detectadas y supone que la madre esté accesible a las mismas; 2) la interpretación correcta de las señales, para evitar las distorsiones y que la madre empatice con el infante; 3) la respuesta adecuada de la madre, la cual debe estar acorde a la etapa evolutiva del niño/a y a la situación que ocurre, previendo así una contingencia en la respuesta y 4) la prontitud de la respuesta, lo cual supone poca demora frente a las demandas del niño/a, respetando así sus estados de ánimo y ritmos (Ainsworth, 1969).

En este sentido, una de las suposiciones más importantes es que el estilo de apego que desarrolle el niño(a) dependerá, en gran medida, de la capacidad de la madre para responder a las señales de su hijo/a (Ainsworth et al., 1978; Atkinson et al., 2000; Bowlby, 1969/1982; De Wolff \& van IJzendoorn, 1997; Marrone, 2001). Es decir, la conducta sensible de la madre promoverá y permitirá que el niño/a organice sus conductas de exploración del entorno y de cercanía cuando sienta alguna amenaza, lo cual ha sido denominado conducta de base segura (Bowlby, 1969/1982; Waters \& Cummings, 2000). Estos patrones de interacción con la madre se incorporan en lo que Bowlby denominó modelos operativos internos. Así, las representaciones mentales de un niño cuya madre haya mostrado disponibilidad y respuestas congruentes con sus necesidades considerarán a las figuras de cuidado como fuentes de protección (Atkinson et al., 2005; Román, Hodges, Palacios, Moreno, \& Hillman, 2018).

A partir de la descripción de Ainsworth (1969) de la calidad de la respuesta de la madre y su influencia en el apego seguro, diversos estudios y meta-análisis han evidenciado la relación entre ambos constructos reportando correlaciones significativas que varían en el tamaño del efecto (Atkinson et al., 2000; Bakermans-Kranenburg, van IJzendoorn, \& Juffer, 2003; Bigelow et al., 2010; De Wolff \& van IJzerdoorn, 1997; Nievar \& Becker, 2008; Zeegers, Colonnesi, Stams, \& Meins, 2017). La fortaleza de estas asociaciones dista de la reportada por el estudio original de Ainsworth (1969), la cual es comparativamente más alta en todos los casos. Es por ello que se han considerado posibles explicaciones a estas diferencias, como la diversidad y variantes de la conceptualización de los constructos y los métodos empleados para evaluarlos (Maia \& Veríssimo, 2012; Nievar \& Becker, 2008; Posada et al., 2013; Román et al., 2018). Algunos estudios mencionan, por ejemplo, que la sensibilidad implica que la madre esté involucrada con su hijo/a, pero que este involucramiento en sí mismo no diferencia a los niños/as con apego seguro de los de apego evitativo. No obstante, se coincide en posicionar a la sensibilidad de la madre como elemento central para promover el apego seguro (De Wolff \& van IJzendoorn, 1997; van den Boom, 1997; van IJzendoorn, 1995) y ser, de esta manera, una variable para intervenir en incrementar la seguridad en los niños y niñas (Juffer, Bakermans-Kranenburg, \& van IJzendoorn, 2009). Incluso, algunos teóricos del apego han llamado a esta relación la hipótesis de sensibilidad (van IJzendoorn \& Sagi-Schwartz, 2008) y en el contexto latinoamericano se ha 
llegado a encontrar valores de hasta .61 (Nóblega, 2012; Posada et al., 1999; Posada et al., 2002; Posada, Carbonell, Alzate, \& Plata, 2004), demostrándose, de alguna manera, la universalidad de esta relación (Posada, 2013).

Respecto a la evaluación de la sensibilidad, existe aún discusión entre los teóricos del apego acerca de los alcances de la conceptualización del constructo y las formas de evaluación en diferentes contextos (Bretherton, 2013; Pederson, Bailey, Tarabulsy, Bento, \& Moran, 2014; Shin, Park, Ryu, \& Seomun, 2008). Esto se debe, en parte, a la discrepancia entre lo reportado en el estudio inicial de Ainsworth (1969) y los resultados de posteriores investigaciones sobre el tema. Un elemento importante que mencionaron Pederson et al. (1990) es que en el estudio de Ainsworth las descripciones sobre sensibilidad de las madres se basaron en más de 60 horas de observación en contextos naturales durante el primer año de vida del niño/a. De esas observaciones se construyó su instrumento conocido como las Escalas de Sensibilidad Materna (Scales of Maternal Sensitivity), el cual contenía escalas bipolares de sensibilidad vs insensibilidad, cooperación vs interferencia, aceptación vs rechazo y accesibilidad vs postergación, y que evaluaban en un rango de 9 puntos estas polaridades.

El procedimiento observacional demanda una gran cantidad de tiempo y de capacitación para una adecuada observación, que no sería posible de aplicar con el tipo de vida que llevan las familias en la actualidad (Pederson et al., 1990; Pederson et al., 2014). Por ello, Pederson y colegas (1990) elaboraron una medida del comportamiento materno utilizando la metodología Q-Sort (MBQS - Maternal Behavior Q-Sort), con la finalidad de capturar aspectos centrales de sensibilidad en la relación madre-hijo, basándose en conductas específicas y no en componentes globales. De esta manera, se otorga importancia a las características particulares de la sensibilidad de cada figura materna (Pederson et al., 2014; Posada, 2013). El MBQS describe 90 comportamientos tomando como referencia la escala de sensibilidad de Ainsworth y usando la metodología Q-sort empleada en otros instrumentos de medida de los temas de la teoría del apego como el Attachment Q-Sort (AQS, Waters, 1995; Waters \& Deane,
1985). Este tipo de procedimiento es utilizado en observaciones en contextos naturales para luego realizar una valoración en donde se caracterice a la figura materna en función a sus conductas más resaltantes (Pederson et al., 1990). Cabe señalar que el MBQS considera la observación de la madre en momentos en que tiene otras demandas cotidianas además de las del cuidado del infante, debiendo dividir su atención entre las necesidades del niño y las propias (Pederson \& Moran, 1995a). Un comportamiento característico es puntuado con una valoración alta y uno no característico con una baja. Posteriormente, los valores en cada comportamiento son contrastados con el puntaje prototípico de sensibilidad ideal pudiendo obtenerse puntajes que van de -1 a 1 (Behrens, Hart, \& Parker, 2012; Behrens, Parker, \& Kulkofsky, 2014; Pederson \& Moran, 1995a). Este criterio ideal es el resultado de validado en términos de contenido por psicólogos especialistas en desarrollo y apego (Pederson et al., 1990).

En su estudio inicial sobre el instrumento Pederson et al. (1990) plantean una validez convergente con un valor de .90 con las escalas de sensibilidad de Ainsworth, así como confiabilidad inter-evaluador de .75. En los siguientes estudios realizados por los mismos autores esta confiabilidad inter-evaluador aumentó a .94 y .95 en dos períodos de tiempo distintos (Pederson \& Moran, 1995a) y a .91 en otro estudio (Pederson, Gleason, Moran, \& Bento, 1998). Además, en los estudios introductorios del instrumento se reporta relación modesta con el Q-sort del apego, encontrando valores que oscilaron entre .29 y .52 (Moran \& Pederson, 1999), tal como se reporta en otros estudios con instrumentos alternativos de sensibilidad materna (De Wolff \& van IJzerdoorn, 1997) y brindando, de esta manera, evidencia para la validez predictiva del instrumento (Pederson \& Moran, 1996). Cabe resaltar que los autores enfatizan que el MBQS logra discriminar, a madres de niños con apego seguro de los inseguros, usando el AQS para medir la seguridad del vínculo de apego en el niño (Pederson et al., 1990).

Así mismo, es importante mencionar que con el transcurso del tiempo se han ido revisando e implementando diversas versiones del MBQS para incorporar mejoras o adaptarlo a las situaciones naturales de observación en casa 
(Bailey, Moran, Pederson, \& Bento, 2007; Pederson et al., 1990; Pederson \& Moran, 1995a), a la forma de acceder a las conductas mediante videos (Pederson et al., 2014; Tarabulsky et al., 2009) o a edades específicas como los preescolares (Behrens et al., 2014; Posada, Kaloustian, Richmond, \& Moreno, 2007). De este modo, si bien la mayoría de versiones ha usado un único factor para describir la sensibilidad de la madre (Moran, Pederson, Pettit, \& Krupka, 1992; Pederson, Moran, \& Bento, 1999), también se han realizado esfuerzos por encontrar diversas áreas o factores en un análisis más profundo de la sensibilidad (Bailey et al., 2007; Posada et al., 2002).

Así, se encuentra la versión original del MBQS (Pederson et al., 1990) como las versiones mejoradas 2.1 (Pederson \& Moran, 1995a) y 3.1 (Pederson et al., 1999) todas ellas con 90 ítems; la versión de 72 ítems (Moran, Pederson, \& Bento, 2010), otra de 72 ítems con variaciones para el procedimiento de la situación extraña (Behrens, Parker, \& Haltigan, 2011) y la versión corta de 25 ítems creada a partir del MBQS 3.1 cuando se tienen breves períodos de observación (Tarabulsky et al., 2009).

La versión más reciente MBQS 3.1 muestra ventajas importantes respecto a otros instrumentos y a versiones anteriores del instrumento. Como se mencionó, anteriormente, la metodología Q-sort permite identificar la configuración de conductas de la madre, brindando puntajes continuos, evitando sesgos con una distribución fija y detectando grupos de factores característicos (Bailey et al., 2007; Behrens et al., 2014). Así mismo, esta versión tiene como ventaja, respecto a las anteriores, que pretende identificar a las madres ambivalentes de las evitativas $\mathrm{y}$, además, pone énfasis en las conductas directas de la relación con el infante brindando perfiles de madres para indagar en aspectos específicos de la sensibilidad de acuerdo a los postulados de Ainsworth (Bailey et al., 2007; Pederson et al., 1999).

El MBQS ha demostrado adecuada consistencia entre distintas muestras (Atkinson et al., 2005), estabilidad en el tiempo (Pederson \& Moran, 1996; Tarabulsky et al., 2005) y ha sido ampliamente reconocido a nivel internacional, usándolo y validándolo en distintos contextos
(Atkinson et al., 2000, Behrens et al., 2014; Posada et al., 2007; van IJzendoorn, Vereijken, Bakermans-Kranenburg, \& Riksen-Walraven, 2004). De este modo, se reportan confiabilidades superiores a .80 en la mayoría de estudios (Atkinson et al., 1999; Bailey et al., 2007; Tarabulsy et al., 2005).

En Latinoamérica se han usado las versiones 2.1 (Posada et al., 2002; Posada et al., 2004) y de preescolares, en donde se hallan también diferentes dimensiones de la sensibilidad (Posada et al., 2007). En el Perú, existe evidencia sobre el uso del MBQS con preescolares observándose una buena confiabilidad inter-evaluador (Nóblega, 2012). Sin embargo, son pocos los estudios que han tenido participantes menores de tres años (Farkas et al., 2015; Nóblega et al., 2016), siendo las madres de infantes un sector importante de la población a evaluar, particularmente en aquellas de nivel socioeconómico bajo. A partir de lo descrito anteriormente, se propone analizar la pertinencia psicométrica del MBQS 3.1 en el contexto peruano, específicamente la evidencia de validez convergente con la versión MBQS 2.1, y la de criterio, considerando el constructo de apego medido con el AQS.

\section{Método}

\section{Participantes}

Participaron 27 madres y sus infantes de ocho a once meses de edad ( $M=9.04, D E=.98), 10$ niñas (37\%) y 17 niños (63\%). El promedio de edad de las madres fue de 27.96 años $(D E=6.02)$. Todas ellas tenían entre uno y cinco hijos, cuyas edades fluctuaban entre los 8 meses y 20 años. En relación a su nivel educativo, el 63\% tenían estudios secundarios y el $37 \%$ educación superior técnica. Asimismo, en el momento del estudio el $81.5 \%$ realizaba las labores del hogar, mientras que el $18.5 \%$ tenía un trabajo remunerado. Todas las participantes pertenecían a un nivel socioeconómico (NSE) bajo a excepción de dos familias $(7.4 \%)$ que se ubicaban en un NSE medio. En todos los casos la cuidadora principal era la madre.

Para la selección de participantes se realizaron visitas en un centro público de salud de acuerdo a la accesibilidad de las investigadoras. Se seleccionó a madres con hijos entre 8 y 11 meses 
y de nivel socioeconómico bajo y medio. Aquellas madres con hijos menores fueron invitadas posteriormente al estudio, cuando sus hijos cumplieron los 8 meses de edad. Así mismo se excluyeron a potenciales participantes que tuvieran hijos o hijas con alguna patología grave de desarrollo o de salud (i.e. retardo mental, autismo, síndrome de Down u otro síndrome del desarrollo, o prematuridad), de acuerdo al reporte de la madre. En cuanto a las consideraciones éticas, se presentó el objetivo del estudio a todas las interesadas explicando las actividades del mismo y asegurando la confidencialidad y anonimato de los datos. Las madres aceptaban su participación en el estudio al firmar el consentimiento informado correspondiente.

\section{Medición}

La sensibilidad materna fue evaluada mediante el Maternal Behavior Q-Sort (MBQS) en su versión 2.1 (Pederson \& Moran, 1995b) y 3.1 (Pederson et al., 1999), elaborado por Pederson et al. (1990). Este instrumento consta de noventa conductas que describen características generales de la calidad del comportamiento materno.

El MBQS brinda tres modalidades de aplicación: a través de la observación directa de la interacción madre-infante (Pederson \& Moran, 1995a), el auto-reporte que realiza la madre de su propio comportamiento y finalmente el reporte de ideales (Carbonell, Plata, \& Alzate, 2006). En el presente estudio se utilizó la modalidad de observación.

El nivel de sensibilidad se obtiene a través de la distribución de los ítems en función a si estos representan conductas que se consideren como características, o no, de la madre. Las conductas no observadas o aquellas que se manifiestan de igual manera en la madre son colocadas en el centro de la distribución categorizándolas así como ni características ni no características (Pederson \& Moran, 1995b). Los comportamientos clasificados en cada una de estas categorías son divididos en tres subgrupos, obteniéndose finalmente una distribución de nueve categorías que van desde lo más característico de la madre a lo menos característico (Pederson et al., 1999). Mediante un procedimiento estandarizado de calificación cada categoría finalmente está conformada por 10 conductas, a las cuales se les asigna una puntuación del 1 al 9. Así, aquellas que menos caracterizan a la madre obtienen un puntaje entre 1 y 3 , mientras que las que más caracterizan se puntúan entre 7 a 9. Las conductas que no caracterizan o no se observaron, son puntuadas entre 4 y 6 (Salinas-Quiroz \& Posada, 2015).

Para obtener la puntuación global de sensibilidad se correlacionan los puntajes de los 90 ítems que describen el comportamiento de la madre con el criterio teórico de una madre idealmente sensible Así, la puntuación refleja el grado de similitud entre las conductas de la madre y las conductas que se esperaría que una madre ideal tenga con su hijo (Pederson \& Moran, 1995b). En el presente estudio se contó con un grupo de evaluadoras entrenadas en la calificación con el MBQS, tanto en la versión 2.1 como en la 3.1, observando videos de interacciones madre-infante y manteniendo un nivel de consistencia de .70 .

MBQS 3.1 (Pederson et al., 1999). El MBQS 3.1 brinda tres perfiles de sensibilidad materna de acuerdo a las configuración de conductas específicas: Sensitive vs insensitive (Sensible vs no sensible), Disengaged (Desconectada) y Nonsynchronous (No sincrónica) (Bailey et al., 2007). Cada uno de estos perfiles está compuesto por 20 ítems: las 10 conductas que más y las 10 que menos caracterizarían a una madre con cada uno de ellos, ya sea una madre sensible, desconectada o no sincrónica. En conjunto, los tres perfiles explican el $67 \%$ del total de la varianza.

En cuanto al perfil de madre Sensible se encuentran conductas caracterizadas por la sensibilidad, responsividad y la aceptación de su hijo o hija. Las madres con este tipo de perfil no suelen ser punitivas, vengativas o irritables en sus interacciones con el infante. El perfil Desconectada hace alusión a madres que no llegan a identificar las señales de sus hijos por la falta de atención que les dan, por estar haciendo otras actividades, o al ignorarlas. El perfil $\mathrm{No}$ sincrónica se refiere a madres a quienes se les complica adaptarse al ritmo e intensidad de la interacción en respuesta a las señales del infante (Bailey, Waters, Pederson, \& Moran, 1999; Bailey et al., 2007). Una vez obtenidos los puntajes para cada comportamiento, se correlacionan los 
puntajes con el criterio ideal propuesto (Pederson et al., 1999). El coeficiente encontrado refleja el grado de similitud entre las conductas observadas de la madre y cada perfil (Bailey et al., 2007).

En investigaciones anteriores, la confiabilidad inter-observador ha sido reportada entre .68 y .97 (Ortiz et al., 2013; Pereira et al., 2012). En un $30 \%$ de los casos se asignaron dos observadoras quienes realizaron las calificaciones de las familias participantes de manera independiente obteniendo un confiabilidad inter-evaluadora de $.89(D E=.03)$.

MBQS 2.1. (Pederson \& Moran, 1995b). Se usó la versión adaptada del MBQS por Posada et al. (2002) al contexto latinoamericano. Se obtuvieron las puntuaciones y confiabilidades por consistencia interna con el índice alfa de Cronbach para las escalas del comportamiento materno descritas en estudios previos (Nóblega et al., 2016; Posada et al., 2002): (1) Respuesta sensible que a las señales y necesidades del infante ( $\alpha=.88)$; (2) Accesibilidad que refiere a la capacidad de la figura materna para tener en cuenta al niño aun en presencia de otras tareas y actividades $(\alpha=.89)$; (3) Aceptación que se traduce en el tono positivo de la madre en la interacción diaria con el infante $(\alpha=.89)$; (4) Interferencia o falta de apoyo y colaboración en las actividades del niño $(\alpha=.87)$; (5) Actitud activa-animada referida a la participación activa de la madre en la interacción con su hijo $(\alpha=.79)$; (6) Crear un ambiente interesante, es decir, proveer al entorno de estímulos y una organización apropiada para el infante $(\alpha=.80)$ y finalmente, (7) Preocupación por la apariencia física donde la madre muestra atención en que el niño presente una buena apariencia física $(\alpha=.50)$.

En todos los casos se asignaron dos evaluadoras, alternando los pares entre sí, quienes calificaron de manera independiente las grabaciones. Las calificaciones tuvieron un índice de confiabilidad inter-observadora promedio de $.82(D E=.09)$, con un mínimo de 62 y máximo de .92 .

La conducta de base segura, que sirve como constructo de criterio, fue medida con el Attachment Q-Set (Waters, 1995; Waters \& Deane, 1985) el cual fue elaborado como medida para observar los comportamientos entre el niño y la figura materna en contextos naturales. Usa la metodología Q-sort para identificar los comportamientos más característicos de base segura.

AQS (Versión 3, Waters, 1995). Se usó la versión en español de Posada et al. (1999) y adaptada linguísticamente al medio por Nóblega (2012). El AQS presenta 90 tarjetas describiendo conductas del niño, o niña, con su figura materna. Evidencias de la adecuación de las propiedades psicométricas han sido reportadas en diversos estudios (Cassibba, van IJzendoorn, \& D'Odorico, 2000; Nóblega, 2012; Nóblega et al., 2016; Salinas-Quiroz, Morales-Carmona, CruzMartínez, Posada, \& Carbonell, 2014; van IJzendoorn et al., 2004). En todos los casos, los videos de las interacciones registradas en los hogares de las participantes fueron calificados por pares de dos evaluadoras, independientes a la calificación de la conducta materna. El promedio de los índices de confiabilidad inter-evaluadora fue de .83 ( $D E=.07)$, con un rango de .65 a .92 .

\section{Procedimiento}

El presente estudio forma parte de un proyecto multicéntrico el cual tuvo como objetivo explorar el aporte de la sensibilidad materna, las estrategias regulatorias - en especial el uso de la canción de cuna - y las representaciones de apego maternas sobre la seguridad del apego del niño durante el primer año de vida (Nóblega et al., 2016).

De esta manera, en el estudio principal se llevaron a cabo dos reuniones en los hogares de las participantes. En la primera visita se discutió sobre el objetivo y las actividades del estudio, se firmó el consentimiento informado y se recogieron datos sociodemográficos de la familia. Luego se observó y grabó en video la interacción natural entre las madres y sus hijos e hijas en el día por 1.5 horas aproximadamente. En la segunda sesión se aplicaron otras medidas concernientes a las representaciones de apego de la madre, las estrategias de regulación y la seguridad de los niños utilizando procedimientos de reuniónseparación con la madre.

Como una forma de retribuir su participación, se les entregó a las madres un DVD con la filmación de la primera observación y un reconocimiento económico equivalente a 15 dólares. Adicionalmente, las madres tuvieron la 
opción de solicitar una reunión en donde se les ofrecía una orientación psicológica de acuerdo a lo observado en la relación con el niño o dudas respecto a la crianza.

\section{Análisis de datos}

Se utilizó el paquete estadístico IBM SPSS, versión 22, para los análisis respectivos. En primer lugar, se llevaron a cabo análisis descriptivos tanto de las variables principales de estudio como las demográficas. Luego se realizaron los análisis de comparación de dos grupos (i.e. sexo del infante, si hubo separaciones o no de la cuidadora) con la prueba $t$ de Student y de asociación con el índice de correlación $r$ de Pearson.

\section{Resultados}

En la Tabla 1 se aprecian las medidas de tendencia central y de dispersión del MBQS 3.1. Por su parte, la sensibilidad global del MBQS 2.1 presentó un $M=.55(D E=.25)$ con un IC $95 \%$ de $[.45, .64], \quad M d n=.58, \quad \operatorname{Min}=-.02$ y $\operatorname{Máx}=.83$ mientras que la seguridad del apego del AQS mostró un $M=.19$ ( $D E=.25)$, IC $95 \%$ de $[.09, .29]$, $M d n=.20, M i n=-.36$ y Máx=.59.

Tabla 1. Datos Descriptivos del MBQS 3.1

\begin{tabular}{lcccccc}
\hline & $\mathrm{M}$ & $\mathrm{Mdn}$ & $\mathrm{DE}$ & $\mathrm{Min}$ & Máx & IC 95\% \\
\hline Puntaje global & .61 & .67 & .23 & -.04 & .85 & {$[.52, .70]$} \\
Sensible & .76 & .82 & .22 & .04 & .95 & {$[.67, .85]$} \\
Desconectada & -.50 & -.60 & .36 & -.87 & .50 & {$[-.64,-.36]$} \\
No sincrónica & -.44 & -.59 & .35 & -.89 & .44 & {$[-.58,-.30]$} \\
\hline$N=27$ & & & & & &
\end{tabular}

Tabla 2. Correlaciones entre Perfiles de Sensibilidad

\begin{tabular}{lcccc}
\hline & $\begin{array}{c}\text { Puntaje } \\
\text { global }\end{array}$ & Sensible & Desconectada & $\begin{array}{c}\text { No } \\
\text { sincrónica }\end{array}$ \\
\hline Puntaje global & - & $.92 * *$ & $-.95 * *$ & $-.91 * *$ \\
Sensible & & - & $-.84 * *$ & $-.77 * *$ \\
Desconectada & & & - & $.85^{* *}$ \\
No sincrónica & & & & - \\
\hline$* * p<.001$ & & & &
\end{tabular}

Se identificó una asociación positiva entre la edad de la madre y el perfil sensible $(r=.41$, $p=.03)$ y una tendencia con la sensibilidad global $(r=.36, p=.063)$. No se encontraron diferencias en la sensibilidad global ni en ninguno de los perfiles del MBQS 3.1 según sexo o si se dieron separaciones, o no, entre la madre y el infante.
En cuanto a la evidencia de validez basada en la convergencia con la versión previa del MBQS, el puntaje global de sensibilidad del MBQS 3.1 se asoció positiva y significativamente con el del MBQS 2.1 ( $r=.76, p<.001)$.

Tabla 3. Correlaciones entre Perfiles 3.1 y Dimensiones 2.1 de Sensibilidad Materna

\begin{tabular}{|c|c|c|c|c|}
\hline & MBQS 3.1 & Sensible & Desconectada & $\begin{array}{c}\text { No } \\
\text { sincrónica }\end{array}$ \\
\hline MBQS 2.1 & $.76^{* *}$ & $.64 * *$ & $-.78 * *$ & $-.76 * *$ \\
\hline $\begin{array}{l}\text { Respuesta } \\
\text { sensible }\end{array}$ & $.69 * *$ & $.59 * *$ & $-.70 * *$ & $-.64 * *$ \\
\hline Accesibilidad & $.73 * *$ & $.59 * *$ & $-.80 * *$ & $-.65 * *$ \\
\hline Aceptación & $.33^{\mathrm{t}}$ & $.35^{\mathrm{t}}$ & $-.38 *$ & $-.37^{t}$ \\
\hline Interferencia & -.03 & -.02 & .19 & -.15 \\
\hline $\begin{array}{l}\text { Actitud } \\
\text { animada }\end{array}$ & $.51 * *$ & $.41 *$ & $-.61 * *$ & $-.53 * *$ \\
\hline $\begin{array}{l}\text { Ambiente } \\
\text { interesante }\end{array}$ & .32 & .16 & $-.39 *$ & -.28 \\
\hline $\begin{array}{l}\text { Apariencia } \\
\text { física }\end{array}$ & .13 & .13 & .002 & -.16 \\
\hline
\end{tabular}

Para la evidencia de criterio se encontró una correlación positiva y moderada entre la sensibilidad global del MBQS 3.1 y la seguridad del apego AQS $(r=.44, p<.05)$. Además, el perfil de madre sensible $(r=.43, p<.05)$, desconectada $(r=-.36, p=.06)$ y no sincrónica $(r=-.42, p<.05)$ se asociaron con la seguridad del apego.

\section{Discusión}

El propósito de este estudio fue identificar evidencias de validez convergente y de criterio del instrumento de sensibilidad materna MBQS 3.1 en el contexto peruano. Para ello se utilizó como medidas de comparación a una versión previa del MBQS así como un instrumento de seguridad del apego en la niñez, AQS.

El promedio del puntaje de sensibilidad materna evaluada con el MBQS 3.1 sugiere que las conductas maternas en esta muestra se ajustan, en general, con el criterio teórico de lo que se considera una madre sensible. Esto se observa, además, en los promedios de los perfiles sensible, desconectada y no sincrónica. En cuanto a las variables sociodemográficas, se encontró una asociación positiva y mediana entre la edad de la madre y el perfil materno sensible, así como una tendencia positiva con la sensibilidad global. 
Estos resultados son consistentes con algunas evidencias reportadas en donde el ser madre a temprana edad se asocia con una menor sensibilidad (Wright, Hill, Sharp, \& Pickles, 2018). Por el otro lado, a mayor edad la madre podría también contar una mayor experiencia de cuidado que le permita identificar señales y atender las necesidades del infante de manera consistente. Sería recomendable considerar esta variable en estudios más amplios.

La sensibilidad materna evaluada con el MBQS 3.1 se encuentra asociada con el puntaje de sensibilidad global calificada con la versión previa, el MBQS 2.1 con una fuerza de asociación grande (Cohen, 1988). Este resultado permite afirmar que las conductas que configuran el comportamiento sensible materno son recogidas por ambas versiones de manera similar (Pederson et al., 1999). La fortaleza de las asociaciones entre cada uno de los perfiles de comportamiento materno con el puntaje global del MBQS 2.1 admiten considerar al MBQS 3.1 como una herramienta adecuada de evaluación de las interacciones diádicas madre-hijo y no solo como medición de sensibilidad materna.

Por otro lado, y tal como se esperaba, el puntaje global de sensibilidad del MBQS 3.1 se asoció positivamente con la seguridad de apego del infante, con una fuerza de asociación mediana (Cohen, 1988). Este resultado está de acuerdo con uno de los postulados principales de la teoría de apego el cual señala que es en la interacción diádica donde la figura materna contribuye a que el niño establezca la conducta de base segura (Ainsworth, 1969; Ainsworth et al., 1978; Bowlby, 1969/1982; Bowlby, 1988), aún en poblaciones de riesgo (Moran et al., 1992; van IJzendoorn et al., 2004). Los perfiles sensible y no sincrónica también se asociaron significativamente con la seguridad del infante, mientras que se observó una tendencia en la dirección esperada con el de madre desconectada. En conjunto, estos resultados muestran que el MBQS 3.1 contribuye a identificar patrones de comportamiento materno sensible, evitativo y ambivalente y en qué grado cada uno de ellos se asocia con la configuración de conducta de base segura en la infancia.

En cuanto a las limitaciones del estudio puede indicarse el tamaño reducido de la muestra así como del número de horas de grabación y del porcentaje de videos calificados por dos observadores. Por otro lado, en la mayoría de familias participantes se contó con información sobre el nivel socioeconómico y el grado educativo alcanzado tal como fueron reportados por la madre. A su vez, se puede mencionar la varianza compartida entre medidas al evaluarse tanto la sensibilidad como la conducta de base segura en un mismo evento albeit de manera independiente.

El MBQS 3.1 muestra adecuados indicadores de validez basada en evidencia convergente y de criterio en la muestra de madres. Además, esta versión cuenta con ítems-conductas que resaltan la experiencia directa en la interacción madre-infante y con la configuración de tres perfiles de la interacción madre-infante. Es por todo ello que se considera pertinente al MBQS 3.1, revisada por Pederson et al. (1999), como una medida apropiada de sensibilidad materna en el contexto peruano. Para futuros estudios se recomienda considerar un diseño que permita conocer la pertinencia de los instrumentos en el tiempo y analizar la evidencia de validez basada en la estructura interna del constructo.

\section{Referencias}

Ainsworth, M. D. S. (1967). Infancy in Uganda. Infant care and the growth of love. Baltimore, Estados Unidos: John Hopkins University Press.

Ainsworth, M. D. S. (1969). Object relations, dependency, and attachment: A theoretical review of the infant-mother relationship. Child Development, 40, 969-1025.

Ainsworth, M. D. S., Bell, S. M., \& Stayton, D. (1971). Individual differences in Strange Situation behavior of one-year-olds. En H. R. Schaffer (Ed.), The origins of human social relations (pp. 17-57). Londres, Reino Unido: Academic Press.

Ainsworth, M. D. S., Blehar, M., Waters, E., \& Wall, S. (1978). Patterns of attachment: A psychological study of the Strange Situation. Nueva York, Estados Unidos: Lawrence Erlbaum. 
Atkinson, L., Chisholm, V. C., Scott, B., Goldberg, S., Vaughn, B. E., Blackwell, J., ... Tam, F. (1999). Maternal sensitivity, child functional level, and attachment in Down's syndrome. Monographs of the Society for Research in Child Development, 64(3), 45-66. doi:10.1111/1540-5834.00033

Atkinson, L., Goldberg, S., Raval, V., Pederson, D., Benoit, D., Moran, G., ... Leung, E. (2005). On the relation between maternal state of mind and sensitivity in the prediction of infant attachment security. Developmental Psychology, 41(1), 42-53.

doi:10.1037/0012-1649.41.1.42

Atkinson, L., Niccols, A., Paglia, A., Coolbear, J., Parker, K., Poulton, L., ... Sitarenios, G. (2000). A meta-analysis of time between maternal sensitivity and attachment assessments: Implications for internal working models in infancy/toddlerhood. Journal of Social and Personal Relationships, 17, 791810. doi:10.1177/0265407500176005

Bailey, H., Moran, G., Pederson, D., \& Bento, S. (2007). Understanding the transmission of attachment using variable -and relationshipcentered approaches. Development and Psychopathology, 19, 313-341. doi:10.10170S0954579407070162.

Bailey, H., Waters, C., Pederson, D., \& Moran, G. (1999) Ainsworth revisited: An empirical analysis of interactive behavior in the home, Attachment \& Human Development, 1(2), 191-216. doi:10.1080/14616739900134231

Bakermans-Kranenburg, M. J., van IJzendoorn, M. H., \& Juffer, F. (2003). Less is more: Meta-analyses of sensitivity and attachment interventions in early childhood. Psychological Bulletin, 129(2), 195-215.

Behrens, K., Hart, S., \& Parker, A. (2012). Maternal sensitivity: Evidence of stability across time, contexts, and measurement instruments. Infant and Child Development, 21(4), 348-355. doi:10.1002/icd.1747.

Behrens, K., Parker, A., \& Haltigan, J. (2011). Maternal sensitivity assessed during the Strange Situation Procedure predicts child's attachment quality and reunion behaviors. Infant Behavior and Development, 34, 378381. doi:10.1016/j.infbeh.2011.02.07
Behrens, K., Parker, A. C., \& Kulkofsky, S. (2014). Stability of maternal sensitivity across time and contexts using Q-Sort measures. Infant and Child Development, 23(5), 532541. doi:10.1002/icd.1835

Bigelow, A. E., MacLean, K., Proctor, J., Myatt, T., Gillis, R., \& Power, M. (2010). Maternal sensitivity throughout infancy: Continuity and relation to attachment security. Infant Behavior and Development, 33, 50-60. doi:10.1016/j.infbeh.2009.10.009.

Bowlby, J. (1969/1982). Attachment and loss: Vol. I. Attachment. Nueva York, Estados Unidos: Basic Books.

Bowlby, J. (1988). A secure base: Parent-child attachment and healthy human development. Nueva York, Estados Unidos: Basic Books.

Bretherton, I. (2013). Revisiting Mary Ainsworth's conceptualization and assessments of maternal sensitivityinsensitivity. Attachment \& Human Development, $\quad 15, \quad 460-484$. doi:10.1080/14616734.2013.835128

Carbonell, O. A., Plata, S. J., \& Alzate, G. (2006). Creencias y expectativas sobre el comportamiento materno ideal y real en mujeres gestantes desde un abordaje metodológico mixto. Revista Infancia, Adolescencia y Familia, 1(1), 115-140.

Cassibba, R., van IJzendoorn, M. H., \& D'Odorico, L. (2000). Attachment and play in child care centres: Reliability and validity of the attachment Q-sort for mothers and professional caregivers in Italy. International Journal of Behavioral Development, 24(2), 241-255. doi:10.1080/016502500383377

De Wolff, M. S., \& van IJzendoorn, M. H. (1997). Sensitivity and attachment: A meta-analysis on parental antecedents of infant attachment. Child Development, 68, 571-591.

Farkas, C., Carvacho, C., Galleguillos, F., Montoya, F., León, F., Santelices, M. P., \& Himmel, E. (2015). Estudio comparativo de la sensibilidad entre madres y personal educativo en interacción con niños y niñas de un año de edad. Perfiles Educativos, 37(148), 16-33.

Juffer, F., Bakermans-Kranenburg, M. J., \& van IJzendoorn, M. H. (2009). Attachment-based interventions: Heading for evidence-based 
ways to support families. ACAMH Occasional Papers, 29, 47-57.

Maia, J., \& Veríssimo, M. (2012). Diferentes sistemas de cotação no asct são mais-valia ou fragilidade metodológica? Revista Iberoamericana de Diagnóstico y Evaluación - e Avaliação Psicológica, 33(1), 173-198.

Main, M., \& Solomon, J. (1990). Procedures for identifying disorganized/disoriented infants during the Ainsworth Strange Situation. En M. Greenberg, D. Cicchetti \& M. Cummings (Eds.), Attachment in the preschool years (pp. 121-160). Chicago: University of Chicago Press.

Marrone, M. (2001). La teoría del apego: Un enfoque actual. Madrid, España: Lugar Editorial.

Moran, G., \& Pederson, D. R. (1999). From maternal representations to the first relationship by way of maternal sensitivity: Reconceptualization of the developmental model. Psychology Publications, 28. Recuperado de: https://ir.lib.uwo.ca/psychologypub/28

Moran, G., Pederson, D. R., \& Bento, S. (2010). $M B Q S-72$ for detailed video coding: The selected works of Greg Moran. Recuperado de http://works.bepress.com/gregmoran/103

Moran, G., Pederson, D. R., Pettit, P., \& Krupka, A. (1992). Maternal sensitivity and infantmother attachment in a developmentally delayed sample. Infant Behavior and Development, 15, 427-442.

Nievar, M., \& Becker, B. (2008). Sensitivity as a privileged predictor of attachment: A second perspective on De Wolff and van IJzendoorn's meta-analysis. Social Development, 17(1), 102114. doi:10.1111/j.1467-9507.2007.00417.x

Nóblega, M. (2012). Conducta de base segura y sensitividad en niños y madres del distrito de Los Olivos (Tesis de doctorado). Pontificia Universidad Católica del Perú, Lima.

Nóblega, M., Bárrig, P., Conde, G., Nuñez del Prado, J., Carbonell, O. A., Altmann, M., ... Bauer, M. (2016). Cuidado materno y seguridad del apego antes del primer año de vida. Universitas Psychologica, 15(1), 245260. doi:10.11144/Javeriana.upsy15-1.cmsa

Ortiz, J., Nieto, C., Holler, S., Carbonell, O., Plata, T., \& Suarez, L. (2013). Processes evaluation of naturalistic observation and unintentional injury. Preventive intervention in early infancy. International Society for the Study of Behavioral Development, 1(63), 2229.

Pederson, D. R., Bailey, H. Tarabulsy, G., Bento, S., \& Moran, G. (2014). Understanding sensitivity: Lessons learned from the legacy of Mary Ainsworth, Attachment \& Human Development, 16(3), 261-270. doi:10.1080/14616734.2014.900094

Pederson, D. R., Gleason, K., Moran, G., \& Bento, S. (1998). Maternal attachment representations, maternal sensitivity and the infant-mother attachment relationship. Developmental Psychology, 34(5), 925-933. doi:10.1037/0012-1649.34.5.925

Pederson, D. R., \& Moran, G. (1995a). A categorical description of attachment relationships in the mode and its relation to Qsort measures of infant-mother interaction. En E. Waters, B Vaughn, G. Posada, K. KondoIkemura (Eds.), Caregiving, cultural and cognitive perspectives on secure-base behavior and working models: New growing points of attachment theory and research. Monographs of the Society for Research in Child Development, 60(2-3, Serial N²44), 111-132.

Pederson, D. R., \& Moran, G. (1995b). Appendix B. Maternal Behavior Q-set. En E. Waters, B. E. Vaughn, G. Posada, \& K. Kondo-Ikemura (Eds.), Caregiving, cultural, and cognitive perspectives on secure-base behavior and working models: New growing points of attachment theory and research. Monographs of the Society for Research in Child Development, 60(2-3, Serial $\left.N^{\circ} 244\right)$, 247254.

Pederson, D. R., \& Moran, G. (1996). Expressions of the attachment relationship outside of the strange situation. Child Development, 67, 915927.

Pederson, D. R., Moran, G., \& Bento, S. (1999). Manual - Maternal Behaviour Q-sort Version 3.1. Manuscrito sin publicar, Canada: University of Western Ontario, Ontario, Canadá.

Pederson, D. R., Moran, G., Sitko, C., Campbell, K., Ghesquire, K., \& Acton, H. (1990). 
Maternal sensitivity and the security of infantmother attachment: A Q-sort study. Child Development, 61(6), 1974-1983. doi:10.2307/1130851.

Pereira, J., Vickers, K., Atkinson, L., Gonzalez, A., Wekerle, C., \& Levitan, R. (2012). Parenting stress mediates between maternal maltreatment history and maternal sensitivity in a community sample. Child Abuse and Neglect, 36(5), 433-437.

Posada, G. (2013). Piecing together the sensitivity construct: Ethology and cross-cultural research. Attachment \& Human Development, 15, 637656. doi:10.1080/14616734.2013.842753.

Posada, G., Carbonell, O. A., Alzate, G., \& Plata, S. J. (2004). Through Colombian lenses: Ethnographic and conventional analyses of maternal care and their associations with secure base behavior. Developmental Psychology, 40, 323-333.

Posada, G., Jacobs, A., Carbonell, O. A., Alzate, G., Bustamante, M. R., \& Arenas, A. (1999). Maternal care and attachment security in ordinary and emergency contexts. Developmental Psychology, 35, 1379-1388.

Posada, G., Jacobs, A., Richmond, M., Carbonell, O., Alzate, G., Bustamante, M. R., \& Quiceno, J. (2002). Maternal caregiving and infant security in two cultures. Developmental Psychology, 38(1), 67-78.

Posada, G., Kaloustian, G., Richmond, M. \& Moreno, A. J. (2007). Maternal secure base support and preschoolers' secure base behavior in natural environments. Attachment \& Human Development, 9(4), 393-411.

Posada, G., Ting, L., Trumbell, J., Kaloustian, G., Trudel, M., Plata, S. J., ... Lay, K. L. (2013). Is the secure base phenomenon evident here, there, and anywhere? A cross-cultural study of child behavior and experts' definitions. Child Development. doi:10.1111/cdev.12084

Román, M., Hodges, J., Palacios, J., Moreno, C., \& Hillman, S. (2018). Evaluación de las representaciones mentales de apego a través de las historias incompletas: Aplicación española de Story Stem Assessment Profile (SSAP). Revista Iberoamericana de Diagnóstico y Evaluación - e Avaliacao Psicológica, $\quad 46(1), \quad 5-19$. doi:10.21865/RIDEP46.1.01
Salinas-Quiroz, F., Morales-Carmona, F., CruzMartínez, L. M., Posada, G., \& Carbonell, O. (2014). Aproximación psicométrica al uso de los Q-Sorts en contextos de educación inicial. Acta de Investigación Psicológica, 4(2), 15951611.

Salinas-Quiroz, F., \& Posada, G. (2015). MBQS: Método de evaluación para intervenciones en apego dirigidas a primera infancia. Revista Latinoamericana de Ciencias Sociales, Niñez y Juventud, 13(2), 1051-1063. doi:10.11600/1692715x.13235280514

Shin, H., Park, Y., Ryu, H., \& Seomun, G. (2008). Maternal sensitivity: A concept analysis. Journal of Advanced Nursing, 64(3), 304-314. doi:10.1111/j.1365-2648.2008.04814.x

Tarabulsy, G. M., Bernier, A., Provost, M. A., Maranda, J., Larose, S., ... Tessier, R. (2005). Another look inside the gap: Ecological contributions to the transmission of attachment in a sample of adolescent motherinfant dyads. Developmental Psychology, 41, 212-224.

Tarabulsy, G. M., Provost, M. A., Bordeleau, S., Trudel-Fitzgerald, C., Moran, G., Pederson, D. R., ... Pierce, T. (2009). Validation of a short version of the Maternal Behavior Q-set applied to a brief video record of motherinfant interaction. Infant Behavior \& Development, $\quad 32(1), \quad$ 132-136. doi:10.1016/j.infbeh.2008.09.006

van den Boom, D. C. (1997). Sensitivity and attachment: Next steps for developmentalists. Child Development, 68, 592-594. doi:10.1111/j.1467-8624.1997.tb04219.x

van IJzendoorn, M. (1995). Adult attachment representations, parental responsiveness, and infant attachment: A meta-analysis on the predictive validity of the Adult Attachment Interview. Psychological Bulletin, 117, 387403.

van IJzendoorn, M., \& Sagi-Schwartz, A. (2008). Cross-cultural patterns of attachment: Universal and contextual dimensions. En J. Cassidy \& P. Shaver (Eds.), Handbook of attachment. Theory, research and clinical applications (2a ed., pp. 880-905). Nueva York: The Guilford Press.

van IJzendoorn, M., Vereijken, C., BakermansKranenburg, M., \& Riksen-Walraven, M. 
(2004). Assessing attachment security with the Attachment Q Sort: Meta-analytic evidence for the validity of the observer AQS. Child Development, 75(4), 1188-1213. doi:10.1111/j.1467-8624.2010.01451.x

Waters, E. (1995). The Attachment Q-Set (Version 3). En E. Waters, B. Vaughn, G. Posada \& K. Kondo-Ikemura (Eds.), Caregiving, cultural, and cognitive perspectives on secure-base behavior and working models: New growing points of attachment theory and research. Monographs of the Society for Research in Child Development, 60(2-3, Serial $\left.\mathrm{N}^{\circ} 244\right)$, 234246. doi:10.2307/1166181

Waters, E., \& Cummings, E. M. (2000). A secure base from which to explore close relationships. Child Development, 71, 164172.

Waters, E., \& Deane, K. E. (1985). Defining and assessing individual differences in attachment relationships: Q-methodology and the organization of behavior in infancy and early childhood. En I. Bretherton \& E. Waters (Eds.), Growing points of attachment theory and research. Monographs of the Society for Research in Child Development, 50(1-2), 4165.

Wright, N., Hill, J., Sharp, H., \& Pickles, A. (2018). Maternal sensitivity to distress, attachment and the development of callousunemotional traits in young children. The Journal of Child Psychology and Psychiatry, 59(7), 790-800. doi:10.1111/jcpp.12867

Zeegers, M. A., Colonnesi, C., Stams, G. J., \& Meins, E. (2017). Mind matters: A metaanalysis on parental mentalization and sensitivity as predictors of infant-parent attachment. Psychological Bulletin, 143(12), 1245-1272. doi:10.1037/bul0000114 